\title{
Morphological, optical and electrical properties of spray coated zinc ethyl xanthates for decomposition within a poly(3- hexylthiophene-2,5-diyl) matrix
}

\author{
Matthew Bishop ${ }^{1,2}$, Bamidele Akinwolemiwa ${ }^{1,2}$, Lun Zhang $^{2}$, Chuang Peng ${ }^{3}$, Di $\mathrm{Hu}^{1,2,4}$ \\ ${ }^{1}$ International Doctoral Innovation Centre, University of Nottingham Ningbo China, Ningbo, 315100, PR China \\ ${ }^{2}$ Department of Chemical and Environmental Engineering, and Energy Engineering Research Group, \\ Faculty of Science and Engineering, The University of Nottingham Ningbo China, Ningbo 315100, PR China \\ ${ }^{3}$ School of Resource and Environmental Science, Wuhan University, Wuhan 430072, Hubei, PR China \\ ${ }^{4}$ New Materials Institute, The University of Nottingham Ningbo China, Ningbo 315100, PR China
}

\begin{abstract}
This work investigates the deposition of hybrid layers, for next generation in energy conversion, via spray coating. Understanding the effect that this deposition procedure has on these layers could lead to the rapid development of these technologies, for both laboratory applications and commercialisation. Synthesised zinc ethyl xanthate and poly(3-hexylthiophene-2,5-diyl) was spray-coated on substrates and heated to a temperature of $160{ }^{\circ} \mathrm{C}$, to the hybrid film. Optical, morphological and conductive properties of these films were investigated and linked to the spray coating duration. It was revealed that shorter-duration spray times led to relatively low conductivity and smoother films, moreover, an increase in spraying duration also led to an increase in conductivity, but with increased roughness, from $6.178 \mathrm{~nm}$ to $8.317 \mathrm{~nm}$. As the spray time was further increased factors, including film layering effects, led to a gradual decrease in conductivity accompanied by a decrease in the roughness. Smoother films were shown to result in higher light absorption, characterised by wider band gaps, which could be due to the crystal structure of the inorganic phase. The controllability of this rapid, facile, and inexpensive spray deposition process was then demonstrated in fabrication of prototype photovoltaic devices.
\end{abstract}

\section{Introduction}

Thin film metal chalcogenide/organic materials have been extensively studied in photovoltaics [1-5]. These active layers can be deposited through various methods, including spin coating [5-7] and spray coating [5, 7]. Within each of these deposition procedures there are many macroscopic parameters to be controlled, each of which alter structural features, such as thickness and roughness, of the deposited active layer. Spin coating deposition is a very popular technique, however, it is a very complex and time consuming technique $[8,9]$. Spray coating is a very useful technique for high speed synthesis of active layers for laboratory applications while allowing for easy scale-up to commercialisation. The current understanding, however, of the growth of thin films during spray deposition is low, and it is important to better understand how the spray coating process can be modified to have a higher level of control on the morphology of the active layers to further improve the applicability of this technique [10].

Bulk Heterojunctions (BHJs) refer to multiple phases that are mixed within one active layer, and have been deposited using both spin and spray coating techniques $[4,5,7,11,12]$, thus creating a three-dimensional blend of electron-donating and electron-accepting materials, which in turn offers a large surface area and can increase exciton dissociation [10]. Organic solar cells are highly can typically be produced at a lower cost than their inorganic counterparts, while being mechanically flexible with a comparatively lower negative impact on the environment, however, these organic systems usually have lower efficiencies and a reduced mechanical stability. BHJs comprising of both organic and inorganic components have been shown to combine their respective advantages, whilst synergistically reducing some of the disadvantages [13-18]. Therefore, it has been demonstrated that it is desirable to combine both organic and inorganic materials.

This work focuses on the synthesis of zinc sulphide, a low toxicity, wide band gap semiconductor $[19,20]$, via decomposition of zinc ethyl xanthate precursors (ZnXan), dispersed in a poly(3-hexylthiophene-2,5-diyl) (P3HT) matrix using spray coating deposition, without using a surfactant layer [21]. Using this single source precursor simplifies the method for producing these active layers, allowing for a one step deposition method [11]. The $\mathrm{ZnXan}$ precursor was selected due to the low temperatures required for decomposition to $\mathrm{ZnS}$, causing little damage to the polymeric component, allowing for this single step deposition [22, 23]. The effects of varying the process conditions, namely spray time, was 
the main investigation undertaken in this work. This was conducted through studying how the variation of the spraying time influences the roughness and thickness of the films. Furthermore, the effects of the variations in the structural features on the resulting optical and electronic properties of the prepared films were also tested by using the films as active layers in prototype hybrid photovoltaic BHJ devices.

\section{Experimental}

\subsection{Synthesis of ZnXan}

The synthetic route followed a similar design proposed by Agrawal et al [11] for the synthesis of cadmium ethyl xanthate, simply replacing cadmium chloride with $0.7 \mathrm{~g}$ of zinc chloride. The off-white precipitate (ZnXan) was washed with de-ionized water, and dried at $50^{\circ} \mathrm{C}$.

\subsection{Synthesis of hybrid BHJ}

The mass of P3HT and ZnXan used was kept constant at $5.0 \mathrm{mg}$ and $62.35 \mathrm{mg}$ respectively, in order to obtain a one to one weight ratio of P3HT:ZnS. These were dissolved into a solution of $5.0 \mathrm{ml} \mathrm{1,2-dichlorobenzene}$ (DCB) with $1.0 \mathrm{ml}$ pyridine.

The spray distance and orientation was kept constant at a horizontal distance of $10 \mathrm{~cm}$, between the nozzle and substrate, held perpendicular to the substrate. The spray time was the only parameter varied, using 10, 15, 20, 25 and 30 seconds (s) as variables. These deposited films were then heated to $160{ }^{\circ} \mathrm{C}$ for 30 minutes to decompose the xanthate.

These samples were then analysed for comparison, noting how changes in this deposition affected the properties of the films.

\subsection{Characterisation techniques}

The thickness and morphology of active layer surfaces were characterised in air using Veeco 3100 scanning probe microscope (SPM).

For ascertaining the optical spectra, the active material on the ITO-glass substrate was tested in a Cary 5000 $\mathrm{UV}$-vis absorption spectrometer at a rate of $400 \mathrm{~nm} / \mathrm{s}$, between $350-750 \mathrm{~nm}$. One cycle was taken for each sample.

The resistive properties of the films were characterised with the aid of AC Impedance Spectroscopy, using the PARSTAT 2000 electrochemical workstation from Princeton Advanced Instruments, at an amplitude of $20 \mathrm{mV}$, using a simple two-point probe method, without light simulation i.e. at dark open circuit voltage $\left(\mathrm{OCV}_{\text {dark }}\right)$, which was at a value of a few millivolts across all the films investigated. The bulk resistance was taken to be the real part of the impedance at $100 \mathrm{KHz}$

\section{Results and discussion}

It was proposed that three different factors could be altered from the deposition parameters:

1. Thin film growth mechanism,

2. Inorganic crystallisation,

3. Degree of order within the polymer.

Scanning probe microscopy (SPM) images of the ZnS:P3HT BHJs were collected at various deposition times. Table 1 shows a summary table of results collected.

Table 1. Summary table of the average results for all samples collected using SPM data.

\begin{tabular}{|c|c|c|c|c|c|}
\hline & $10 \mathrm{~s}$ & $15 \mathrm{~s}$ & $20 \mathrm{~s}$ & $25 \mathrm{~s}$ & $30 \mathrm{~s}$ \\
\hline Thickness $(\mathrm{nm})$ & 36.58 & 47.82 & 65.87 & 49.31 & 39.41 \\
\hline $\begin{array}{c}\text { Roughness root } \\
\text { mean square }(\mathrm{nm})\end{array}$ & 6.18 & 6.89 & 8.32 & 6.80 & 4.74 \\
\hline
\end{tabular}

The surface roughness, see Table $\mathbf{1}$, shows that the longest deposition time displays the smoothest surface, and the middle deposition time displays the roughest surface.
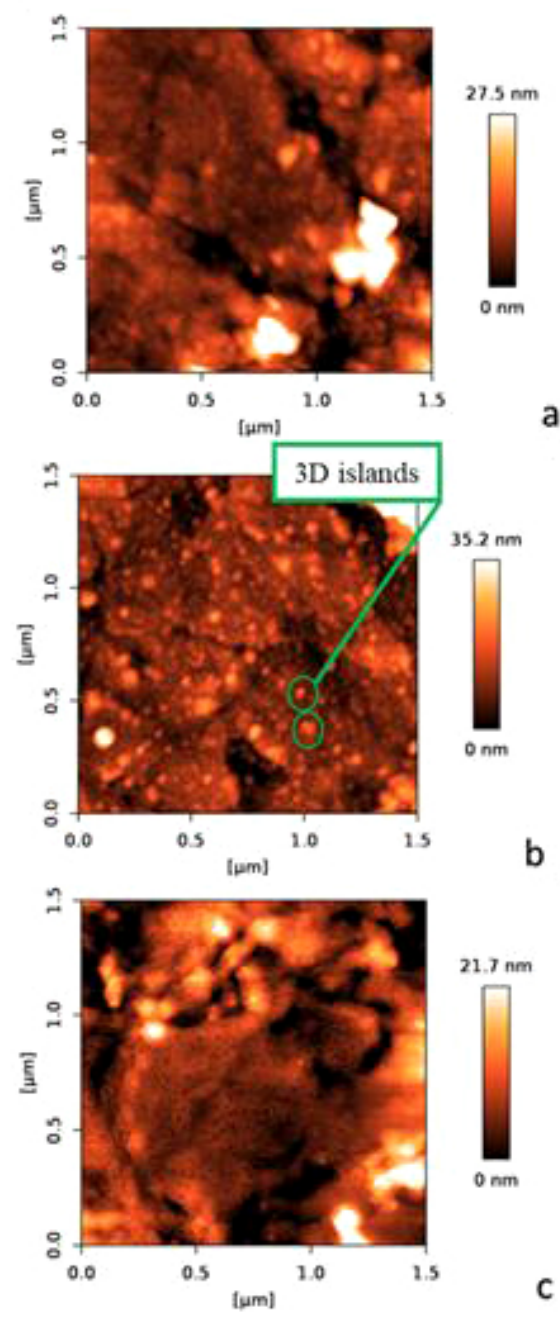

Fig 1. SPM for a) 10 seconds deposition, b) 20 seconds deposition, c) 30 seconds deposition. 
The SPM images, see Fig 1, show the initial growth of a monolayer, before the formation of 3D islands, which in turn forms another monolayer, which suggests that a Stranski-Krastanov thin film growth mechanism might be ascribed to the formation of these films [40]. However, this mechanism does not fully describe the observed film growth as the longest deposition time decreases again in thickness. As previously mentioned, the spray orientation in this work is horizontal. Thus, with an increased amount of deposited precursor, the formation of macroscopic drips is possible, and this could reduce the film thickness whilst also lowering the measured roughness, this would potentially help with a potential photovoltaic device, increasing the Fill Factor as recombination sites are reduced. However, macroscopic drips were not noted during deposition and films remained homogeneous at all deposition times, suggesting another factor is the predominant mechanism in spray deposition.

Average absorption spectra were collected for the varying deposition times, and as the deposition time has an effect on the thickness these spectra were normalised by thickness, see Fig 2.

One key observation is that the spectra follow the same peaks, at roughly $370,520,550$ and $600 \mathrm{~nm}$, with the peak at roughly $600 \mathrm{~nm}$ being attributed to intrachain interactions in P3HT [11]. This would suggest that the chemically active species within the active layers are the same, which is to be expected. The intensity of the peak related to P3HT intra-chain interactions, seen at roughly $600 \mathrm{~nm}$, appears to be more intense for the middle deposition times, thereby suggesting a greater degree of order, which would result in an enhanced conductivity $[25,26]$.

Another key observation relates to the relative magnitude. As can be clearly seen in the graph the samples made from 30 seconds of deposition are the most absorbing. Samples made with 20 seconds of deposition are the least absorbing. This shows an inverse relationship between roughness, recorded from the SPM investigation, see Table 1, and the absorption. A potential reason for this trend in absorption could be related to the light scattering effects, i.e., rougher samples scatter light more, and if more light is scattered on the surface of the layer then less light will be absorbed.

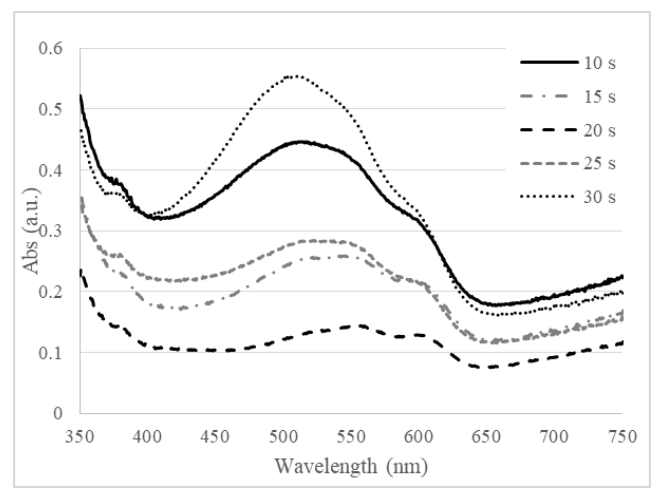

Fig 2. UV-vis absorbance spectra for the varying deposition times.
It was shown in previous works [6,27] that band gap energies can be calculated from Tauc plots. The band gaps, see Table 1, were shown to mimic the same trend as noted from the intensity of the absorption spectra.

Table 2. Calculated band gap values from Tauc plots.

\begin{tabular}{|l|l|l|l|l|l|}
\hline $\begin{array}{l}\text { Deposition } \\
\text { time (seconds) }\end{array}$ & 10 & 15 & 20 & 25 & 30 \\
\hline Band gap (eV) & 2.23 & 2.12 & 2.09 & 2.14 & 2.24 \\
\hline
\end{tabular}

It has been shown that the lattice parameter is inversely proportional to the band gap [28], however, due to the nature of the active layer, x-ray diffraction (XRD) analysis could not be conducted. Since the active layer is primarily $\mathrm{P} 3 \mathrm{HT}$, with isolated nanocrystals of $\mathrm{ZnS}$, effective spectra cannot be collected as the level of crystallinity is too low. Though, it is worth noting that the variation in band gap is quite small, and might not significantly alter the performance of the device.

As conjugated polymers use their macromolecular chains to facilitate the movement of delocalised charge. It has been well-established that both structural and morphological properties can contribute to the mobility of charge in conjugated polymers, particularly crosslinks, loops, kinks and crystal structure $[25,26]$. Therefore, it is expected that the morphological changes, induced through the variation in deposition time, would alter the electrical properties of the produced films. As seen in Fig 3, which subsumes the thickness, an obvious trend can be seen that clearly correlates the roughness with conductivity.

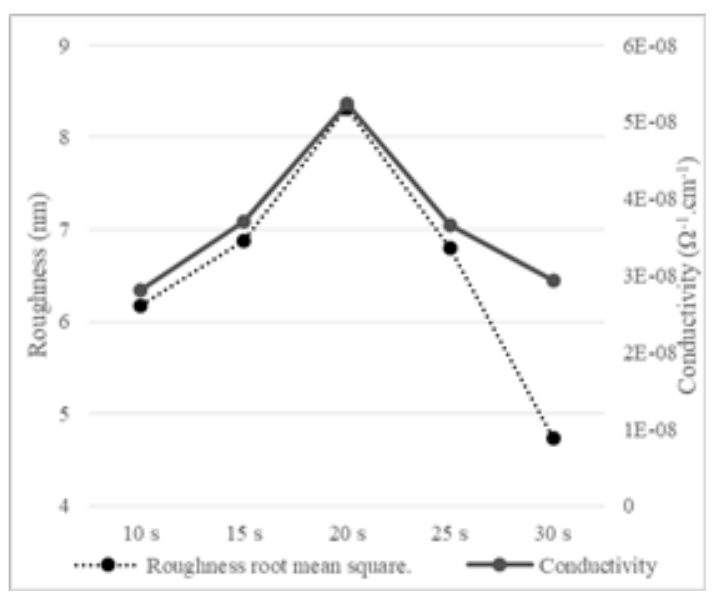

Fig 3. Correlation of roughness and conductivity with varying deposition time.

The degree of order in a polymer is expected to have varying effects on the conductivity of a film, since their electrical conductivity has been described through the so-called hopping mechanisms either within the backbone or across the interlinkage between macromolecules [29]. Therefore, higher conductivity is achievable with morphological that could allow for a relatively ordered macromolecular backbones. This obviously correlates with the surface roughness of the 
films due to the interrelationship between roughness and conductivity, see Fig 3. The conductivity is therefore indicative of how the polymer chain is orientated, which varies with varying spray deposition parameters. This shows that the deposition time has an effect on the formation of the nanostructure of the film, which in turn defines the degree of order for the P3HT deposited. Essentially, the varying degree of roughness could be correlated with the alignment of the P3HT chains, as a result of the film growth mechanism, which would affect the transport of charge within the active layer.

This could imply that the nucleation mechanism resulting in the film formation for spray-coating duration of 20 seconds provides energetic effects that are suitable for separation of the polymer chains, providing a more ordered structure, which is also reflected in the absorbance spectra, see Fig 2. Further increasing deposition time, and therefore time of nucleation, might result in the formation of detrimental cross-linkages, via $\mathrm{ZnS}$ nanoparticles, that could increase the transport pathlength. Short duration spraying, on the other hand, would have insufficient time for the formation of separation, which could effectively lead to dead-ends [25].

\section{Prototype photovoltaic device}

In this section the potential application of this active layer in photovoltaic devices is demonstrated. These BHJs were spray-coated on ITO substrates, and then decomposed at $160{ }^{\circ} \mathrm{C}$ for 30 minutes. Finally, a gold electrode was deposited via sputter coating. The currentvoltage (I-V) curves were calculated (measured using $\mathrm{CHI}$ 660C from $\mathrm{CH}$ Instruments) at ambient conditions, under one sun illumination $\left(100 \mathrm{~mW} / \mathrm{cm}^{-2}\right)$, key photovoltaic information is presented in Table 3 and I-V curves are presented in Fig 4.

Table 3. Photovoltaic feature of ITO-ZnS/P3HT-Au solar cells, with bulk heterojunctions deposited via spray coating at 10,20 and 30 seconds.

\begin{tabular}{|l|l|l|l|}
\cline { 2 - 4 } \multicolumn{1}{c|}{} & $10 s$ & $20 s$ & $30 s$ \\
\hline$V_{O C}(\mathrm{~V})$ & 0.236 & 0.224 & 0.256 \\
\hline$J_{S C}\left(\mathrm{~mA} / \mathrm{cm}^{2}\right)$ & 0.033 & 0.129 & 0.040 \\
\hline Fill Factor & 0.622 & 0.495 & 0.637 \\
\hline PCE $(\%)$ & 0.004831 & 0.01436 & 0.006552 \\
\hline
\end{tabular}

Photovoltaic features of fabricated devices are reported in Table 3. As shown Jsc and the FF values all peak at 20 seconds of deposition, while the Voc and PCE is at the lowest value at 20 seconds of deposition. 20 seconds produced the highest Jsc observed due to favourable transport mechanism as confirmed by the electrical parameters, as demonstrated in Fig 3. Voc values stays at roughly $0.24 \mathrm{~V}$ for all deposition variations, which demonstrates little change to chemical characteristics with varying deposition parameter, as previously displayed in Fig 2, the minor variation is likely due to the varying band gaps, see Table 2 , as these two characteristics have been shown to be interlinked. A significantly lower FF was observed for samples deposited at 20 seconds, indicating a diminished maximum power of the device. This is likely due to increased recombination of excitons at inorganic grain boundaries, attributed to the increase in surface roughness and noted 3D islands, see Fig 1. However, due to increased conductivity seen from films deposited at 20 seconds, see Fig 3, it was shown that devices deposited at 20 seconds vastly outperformed their counterparts. This suggests that the increased conductivity enhances the improved electron-hole transport in the device despite higher recombination.

From the features of the I-V curves, the maximum power point of the cells corresponds to the effect of the bulk resistivity and the absorption of the film. As was observed from Fig 4, the $20 \mathrm{~s}$ spraying time resulted in lower power compared to either 10 or $30 \mathrm{~s}$, despite its conductivity been the highest. Therefore, the film morphology obtained at different spraying times is reflective of a combined connectivity between the active layer and the substrate, formed from the thin film growth mechanism and polymer chain order. Good connectivity between all the components would translate to higher power of the devices, however, the displayed power can be limited by the interconnection between the components of the active layer.

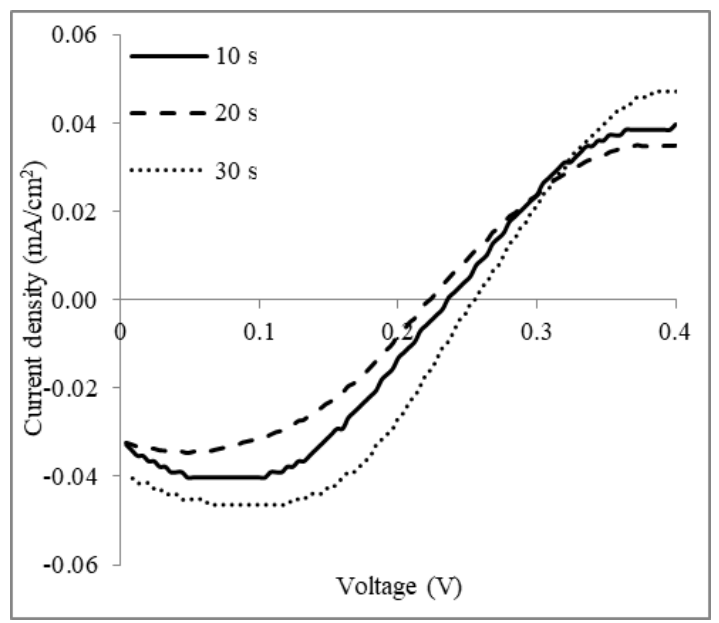

Fig 4. IV curves of ITO-ZnS/P3HT-Au with bulk heterojunctions deposited via spray coating at 10, 20 and 30 seconds.

The deposition time would, in practice, relate to the time required for various nucleation and combination process of the film to form on the substrate. Therefore, the measured photovoltaic properties reflect the ability of the spraying time to induce requisite power performance. It is suggested that the film formation process, is governed by a layering effect, which is a combination of thin film growth and polymer chain order, related to the time of spraying.

\section{Conclusions}

Structural, electrical and optical properties of in-situ synthesised ZnS:P3HT bulk heterojunctions prepared 
through spray coating were investigated in this article. The potential of the as-prepared films for photovoltaic applications were demonstrated, investigating how the deposition time and the effect this had on the microscopic features of the active layers. It was shown that short duration spray times lead to low order in P3HT chain, resulting in lower conductivity. As the spraying time increases there is an increased order of polymer backbones, allowing for the formation of optimal amounts of interlinkage and cross-linkage leading to the highest measured conductivity, while also increasing roughness and decreasing absorbance. As spray times increased further, additional factors, such as detrimental cross-linkage, came into play, resulting in lower conductivity films with decreased roughness and higher absorbance. It has also been shown that the smoother samples yield larger optical band gaps, which might be due to the crystal structure of the inorganic phase, though this could not be verified due to overwhelming amorphous features within deposited films. Photovoltaic devices assembled using active films prepared with these deposition parameters showed that as the conductivity of the materials increases the Jsc values also increase, however, the FF is dramatically reduced, which is suggested to be due to the increased recombination of excitons from inorganic grain boundaries. Furthermore, the observed surface roughness can also be linked to various layering effects which could be indicative of varying connectivity between the components of the deposited films.

This work received financial support from the Provincial Applied Research Programme for Commonweal Technology (2017C31104), and the National Natural Science Foundation of China (21503246). The author acknowledges the financial support from the International Doctoral Innovation Centre, Ningbo Education Bureau, Ningbo Science and Technology Bureau, and the University of Nottingham. This work was also supported by the UK Engineering and Physical Sciences Research Council [grant numbers EP/G037345/1 and EP/L016362/1].

\section{References}

1. M. Bredol, K. Matras, A. Szatkowski, J. Sanetra, A. Prodi-Schwab, Sol. Energy Mater. Sol. Cells 93 662666 (2009)

2. R.J. Zhou, J.G. Xue, ChemPhysChem, 13 2471-2480 (2012)

3. S.L. Zhao, H.A. Wang, Y. Zhou, L. Liao, Y. Jiang, X. Yang, G.C. Chen, M. Lin, Y. Wang, H.L. Peng, Z.F. Liu, Nano Res. 8 288-295 (2015)

4. N. Kumar, V. Dutta, J. Colloid Interface Sci., 434 181187 (2014)

5. E.A. Lewis, P.D. McNaughter, Z.J. Yin, Y.Q. Chen, J.R. Brent, S.A. Saah, J. Raftery, J.A.M. Awudza, M.A. Malik, P. O'Brien, S.J. Haigh, Chem. Mater. 27 21272136 (2015)

6. A.B. Murphy, Sol. Energy Mater. Sol. Cells., 91 13261337 (2007)
7. C. Girotto, B.P. Rand, J. Genoe, P. Heremans, Sol. Energy Mater. Sol. Cells., 93 454-458 (2009)

8. Q.M. Zhang, M.J. Serpe, ACS Appl. Mater. Interfaces, 7 27547-27553 (2015)

9. H.W. Hsu, C.L. Liu, Rsc Adv. 4 30145-30149 (2014)

10. F. Aziz, A.F. Ismail, Mater. Sci. Semicond. Process., 39 416-425 (2015)

11. V. Agrawal, K. Jain, L. Arora, S. Chand, J. Nanopart. Res., 151697 (2013)

12. M. Tejos, B.G. Rolon, R. del Rio, G. Cabello, Mater. Sci. Semicond. Process., 11 94-99 (2008)

13. M. Wright, A. Uddin, Sol. Energy Mater. Sol. Cells, 107 87-111 (2012)

14. W. Jaimes, G. Alvarado-Tenorio, C. Martinez-Alonso, A. Quevedo-Lopez, H.L. Hu, M.E. Nicho, Mater. Sci. Semicond. Process., 37 259-265 (2015)

15. N.A. Ogurtsov, A.A. Pud, O.P. Dimitriev, Y.P. Piryatinski, P.S. Smertenko, Y.V. Noskov, A.S. Kutsenko, Mol. Cryst. Liq. Cryst., 536 33-40 (2011)

16. F.F. Cao, H. Wang, Z.H. Xia, X. Dai, S. Cong, C. Dong, B.Q. Sun, Y.H. Lou, Y.H. Sun, J. Zhao, G.F. Zou, Mater. Chem. Phys., 149 124-128 (2015)

17. J. Jung, Y.J. Yoon, M. He, Z.Q. Lin, J. Polym. Sci., Part B: Polym. Phys., 52 1641-1660 (2014)

18. S. Obuchovsky, I. Deckman, M. Moshonov, T.S. Peretz, G. Ankonina, T.J. Savenije, G.L. Frey, J. Mater. Chem. C, 2 8903-8910 (2014)

19. U. Jabeen, T. Adhikari, S.M. Shah, D. Pathak, T. Wagner, J.M. Nunzi, Eur. Phys. J. Appl. Phys., 78 (2017).

20. J.P. Borah, J. Barman, K.C. Sarma, Chalcogenide Lett., 5 201-208 (2008)

21. J. Cusack, M.G.B. Drew, T.R. Spalding, Polyhedron., 23 2315-2321 (2004)

22. H.C. Leventis, S.P. King, A. Sudlow, M.S. Hill, K.C. Molloy, S.A. Haque, Nano. Lett., 10 1253-1258 (2010)

23. P.S. Nair, T. Radhakrishnan, N. Revaprasadu, G. Kolawole, P. O'Brien, J. Mater. Chem., 12 2722-2725 (2002)

24. C. Ratsch, J.A. Venables, J. Vac. Sci. Technol. A, 21 96-109 (2003)

25. U. Koldemir, S.R. Puniredd, M. Wagner, S. Tongay, T.D. McCarley, G.D. Kamenov, K. Müllen, W. Pisula, J.R. Reynolds, Macromolecules, 48 6369-6377 (2015)

26. S.D. Oosterhout, M.M. Wienk, S.S. van Bavel, R. Thiedmann, L. Jan Anton Koster, J. Gilot, J. Loos, V. Schmidt, R.A.J. Janssen, Nat. Mater., 8 818-824 (2009)

27. M.A. Buckingham, A.L. Catherall, M.S. Hill, A.L. Johnson, J.D. Parish, Cryst. Growth Des., 17 907-912 (2017)

28. R. Dalven, Phys. Rev. B, 8 6033-6034 (1973)

29. A.N. Aleshin, E.L. Alexandrova, Phys. Solid State, 50 1978-1984 (2008) 